MATEC Web of Conferences 4, 04003 (2013)

DOI: $10.1051 /$ matecconf/20130404003

(c) Owned by the authors, published by EDP Sciences, 2013

\title{
Physico-chemical and membrane-interacting properties of D-xylose-based bolaforms. Influence of the anomeric configuration
}

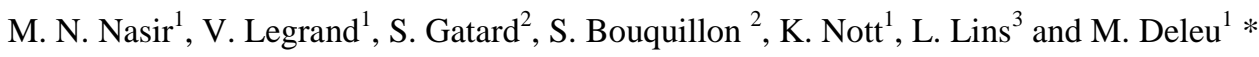 \\ ${ }^{1}$ Unité de Chimie Biologique Industrielle, Université de Liège, Gembloux Agro-Bio Tech, Passage des déportés,2, B-5030 \\ Gembloux, Belgium. \\ ${ }^{2}$ Université de Reims Champagne-Ardenne, Institut de Chimie moléculaire de Reims UMR CNRS 6229, boîte no 44, B.P. \\ 1039, 51687 Reims, France. \\ ${ }^{3}$ Centre de Biophysique Moléculaire Numérique, Université de Liège, Gembloux Agro-Bio Tech, Passage des déportés,2, \\ B-5030 Gembloux, Belgium. \\ *Corresponding author: magali.deleu@ulg.ac.be
}

\begin{abstract}
Sugar-based biosurfactants such as xylose-derived bolaforms have interesting properties, for example high biocompatibility and biodegradability which make them potential useful molecules in the pharmaceutical and cosmetic fields. Until now, no detailed analyses of the physico-chemical properties of these compounds have been undertaken. Two symmetrical D-xylose-based bolaforms were chemically synthesized where the two xylose heads are linked via an acetal link to a hydrocarbon chain containing 18 carbon atoms and an unsaturation. The two bolaforms differ only by their anomeric configuration: $\alpha \alpha$ or $\beta \beta$. The $\alpha \alpha$ bolaform exhibits interfacial properties at the air-water interface which is not the case for the $\beta \beta$. FTIR spectroscopy showed that the interactions between the $\alpha \alpha$ bolaform and POPC, a model phospholipid, involve the carbonyl groups of the phospholipid.
\end{abstract}

\section{Introduction}

During recent years, sugar-based biosurfactants have attracted increasing interest because of their high biocompatibility and biodegradability [1]. Our work has focused on xylose-based bolaforms, a particular class of sugar-based biosurfactants. They are symmetric molecules composed of two polar heads, each consisting by a xylose unit, connected by a hydrophobic carbon chain.

This work studies the influence of the anomeric carbon configuration on the physico-chemical properties of xylose-based bolaforms. For that purpose, two symmetrical bolaforms (XC18 $\alpha \alpha$ unsat and $\mathrm{XC} 18 \beta \beta$ unsat) were chemically synthesized in two steps. In the first, the D-xylose is connected by an acetal link to a hydrocarbon carbon chain containing 10 carbon atoms and a terminal unsaturation. In the second step, two subunits are linked together by metathesis. These two molecules differ only by the configuration of the anomeric carbons which are either $\alpha \alpha$ or $\beta \beta$ (Figure 1).

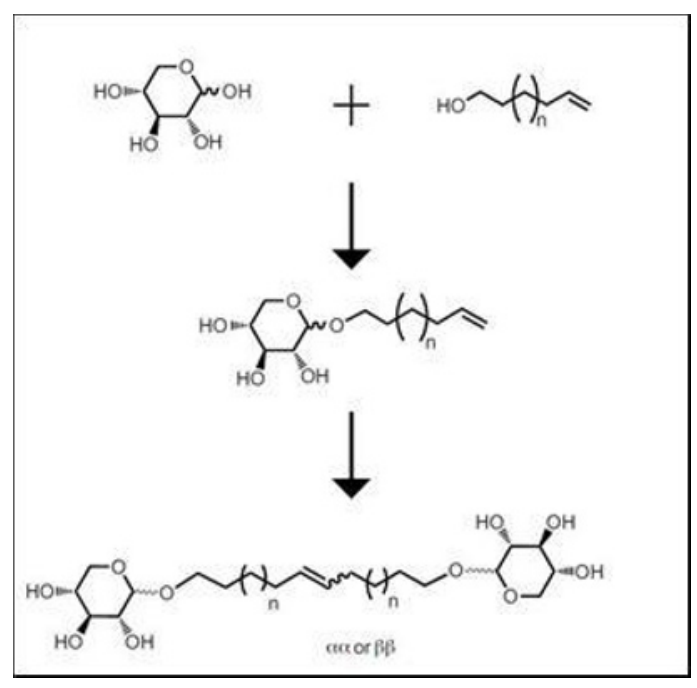

Figure 1: Simplified scheme of the two step-chemical synthesis of the two bolaforms from D-xylose $(n=5)$. XC18 $\alpha \alpha$ unsat $=$ 1-18-bis-octadéc-9-ényl- $\alpha$-D-xylopyranoside; $\mathrm{XC1} 18 \beta \beta$ unsat $=$ 1-18-bis-octadéc-9-ényl- $\beta$-D-xylopyranoside. 
The interfacial properties of these two molecules were initially studied by tensiometry and their critical aggregation concentration (CAC) was determined when pertinent. Then the interactions of XC18 $\alpha \alpha$ unsat with a model phospholipid, 1-palmitoyl-2-oleoyl-sn-glycero-3phosphocholine (POPC), were analyzed in a biomimetic membrane system using FTIR spectroscopy.

\section{Materials and Methods}

\subsection{Chemical Syntheses}

The chemical synthesis of the D-xylose based bolaforms used in this work has been previously reported [1]. The purity of each compound was checked by RPHPLC-ELSD and was found superior to $97 \%$.

\subsection{Tensiometry measurements}

Surface tension was measured with a Tensimat N3 tensiometer equipped with a platinum Wilhelmy plate. All measurements were performed at $25^{\circ} \mathrm{C}$. The bolaforms were dissolved in DMSO and injected into the subphase (ultrapure water) so as to reach different final concentrations ranging from 0.625 to $20 \mu \mathrm{M}$ as described in $[1,2]$.

\subsection{Preparation of multilamellar vesicles (MLV) samples}

Multilamellar vesicles containing POPC or POPC with bolaform were prepared as described in [3].

\subsection{FTIR Spectroscopy}

Infrared spectra were recorded by the means of the Bruker Equinox 55 spectrophotometer after 128 scans at $4 \mathrm{~cm}^{-1}$ resolution. The spectrophotometer was purged with dry air in order to remove any undesired water vapor. All experiments were performed with a demountable cell equipped with $\mathrm{CaF}_{2}$ windows as described in [3]. The spectrum of ${ }^{2} \mathrm{H}_{2} \mathrm{O}$ alone was subtracted from the sample spectrum taken under the same conditions. In the case of MLV spectra, a $20 \mu \mathrm{L}$ sample of the different MLV was deposited on the $\mathrm{CaF}_{2}$ window as described in [3].

\section{Results}

\subsection{Characterization of interfacial properties}

We investigated the interfacial properties of $\mathrm{XC} 18$ $\alpha \alpha$ unsat and XC18 $\beta \beta$ unsat by adsorption experiments at the air-water interface. For each bolaform, Figure 2 gives the evolution of surface pressure as a function of the time after their injection at $2.5 \mu \mathrm{M}$ into the subphase (ultrapure water).

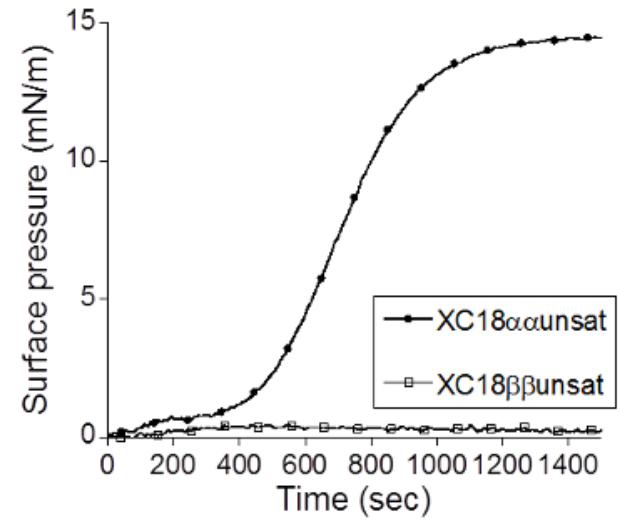

Figure 2: Adsorption experiments of XC18 $\alpha \alpha$ unsat and $\mathrm{XC} 18 \beta \beta$ unsat at the air-water interface monitored by the increase of surface pressure as a function of time. The final concentration of each bolaform in the subphase was $2.5 \mu \mathrm{M}$.

After the injection of the bolaform $\alpha \alpha$, the surface pressure increased before reaching a plateau while no increase of surface pressure was detected with the bolaform $\beta \beta$ even at higher concentration. The modification of the anomeric configuration from $\alpha \alpha$ to $\beta \beta$ for these bolaforms led to a complete loss of surfactant properties. Aggregates were observed in the bulk phase suggesting an insolubilization phenomenon when the anomeric form is $\beta \beta$. We then focused only on the bolaform $\alpha \alpha$. In order to characterize the aggregation behavior of the bolaform $\alpha \alpha$, we injected the molecule at different concentrations and followed the evolution of surface pressure. Figure 3 gives the variation of the equilibrium surface pressure as a function of the concentration in the subphase.

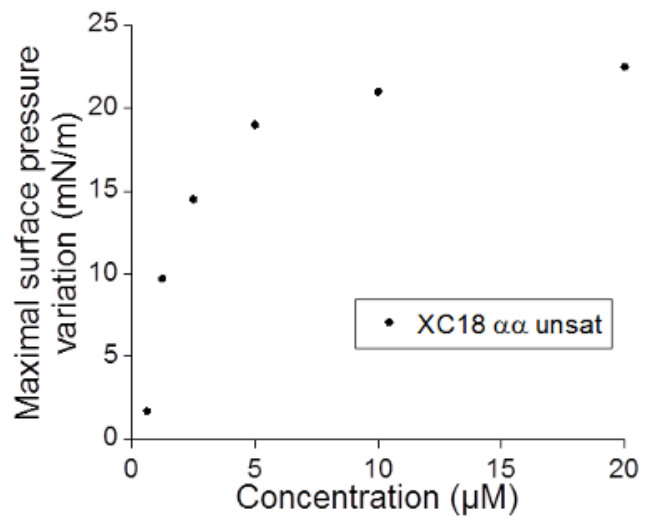

Figure 3 : Maximal surface pressure variation as a function of the concentration of the $\mathrm{XC} 18 \alpha \alpha$ unsat injected into an ultrapure water subphase.

The variation of surface pressure increased with increasing concentrations of bolaform $\alpha \alpha$ and reached a plateau above $5 \mu \mathrm{M}$. From this curve, the critical aggregation concentration of the bolaform $\alpha \alpha$ and the corresponding surface tension, $\gamma_{\mathrm{CAC}}$, was determined to be $4.4 \pm 0.8 \mu \mathrm{M}$ and $54 \mathrm{mN} / \mathrm{m}$, respectively. In comparison to other conventional surfactants [4-6] this CAC value is 
very low. This suggests the high capacity of the bolaform $\alpha \alpha$ to aggregate.

\subsection{Interaction of the bolaform $\alpha \alpha$ with POPC MLVs}

Previous studies reported that XC18 $\alpha \alpha$ unsat was able to penetrate and interact with Langmuir monolayers and bilayers of phospholipids [1,2]. In order to gain more information at the molecular level, we analyzed the interactions of the bolaform $\alpha \alpha$ with POPC in MLVs using infrared spectroscopy. Figure $4 \mathrm{a}$ and $4 \mathrm{~b}$ gives the $1850-1650 \mathrm{~cm}^{-1}$ and $1100-900 \mathrm{~cm}^{-1}$ regions of the infrared spectra of POPC MLVs in the presence or in the absence of XC18 $\alpha \alpha$ unsat, respectively.
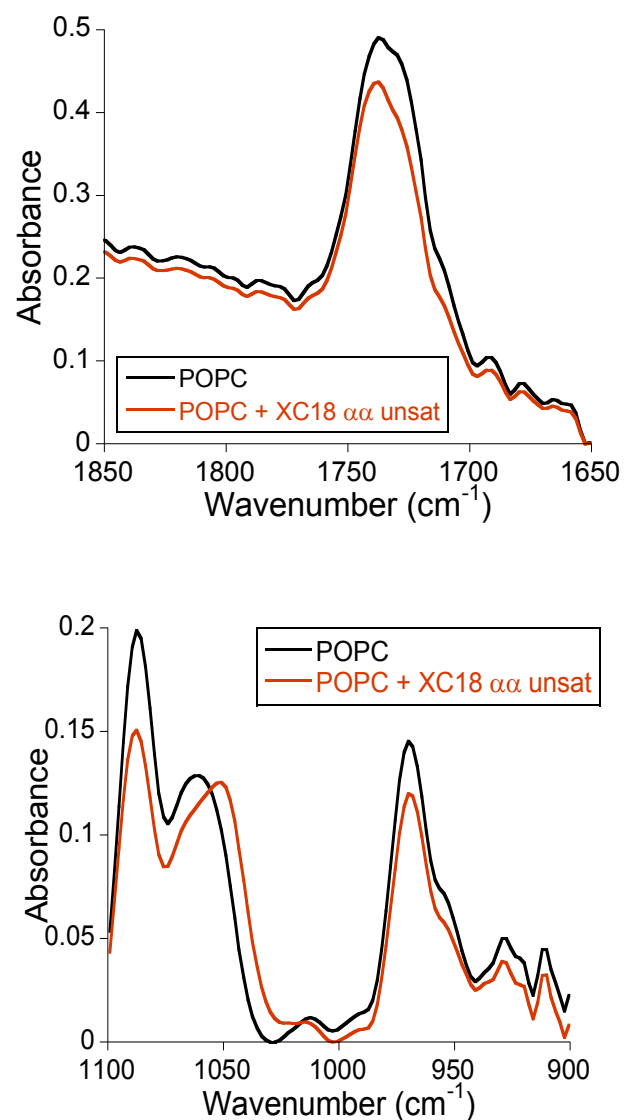

Figure 4: FTIR spectra of the POPC MLVs with or without bolaform XC18 $\alpha \alpha$ unsat. Two regions of the spectra were presented.

In the $1850-1650 \mathrm{~cm}^{-1}$ region, the spectrum of pure POPC MLVs shows a band centered at $1740 \mathrm{~cm}^{-1}$ with a shoulder at $1725 \mathrm{~cm}^{-1}$ which could be attributed to the $\mathrm{C}=\mathrm{O}$ groups of POPC [7-8]. The band at $1740 \mathrm{~cm}^{-1}$ corresponds to the free $\mathrm{C}=\mathrm{O}$ groups while the shoulder at $1725 \mathrm{~cm}^{-1}$ corresponds to the hydrogen-bounded $\mathrm{C}=\mathrm{O}$ ester groups. When the bolaform was inserted into POPC MLVs, the shoulder became less important and shifted slightly to low wavenumber. Even though this change is small, it could suggest an interaction between POPC and bolaform involving $\mathrm{C}=\mathrm{O}$ ester groups. In the 1100-
$900 \mathrm{~cm}^{-1}$ region, the spectrum of pure POPC MLVs shows three major bands located at 1088, 1063 and $970 \mathrm{~cm}^{-1}$. The band at $1088 \mathrm{~cm}^{-1}$ could be attributed to the symmetric stretching vibrations of $\mathrm{PO}^{2-}$ groups of POPC, the band at $1063 \mathrm{~cm}^{-1}$ to the symmetric stretching vibrations of $\mathrm{C}-\mathrm{O}-\mathrm{C}$ bound and the band at $970 \mathrm{~cm}^{-1}$ to the asymmetric stretching vibrations of choline groups [8]. When bolaform $\alpha \alpha$ was inserted into POPC MLVs, the band at $1063 \mathrm{~cm}^{-1}$ was shifted to $1052 \mathrm{~cm}^{-1}$. This suggests that the interactions of the bolaform with POPC MLVs involve the C-O-C groups of the lipid. No change is observed for the bands at $1080 \mathrm{~cm}^{-1}$ and $970 \mathrm{~cm}^{-1}$ suggesting that the choline groups of POPC are not involved in these interactions.

\section{Conclusions}

This work was carried out on a novel series of bolaforms having two D-xylose heads connected to each other with an hydrocarbon chain and differing only by their anomeric carbons which were $\alpha \alpha$ or $\beta \beta$. The interfacial behavior of these two bolaforms was investigated by tensiometry measurements. The bolaform $\alpha \alpha$ was found to have interfacial properties while the bolaform $\beta \beta$ shown none whatsoever, it was located in the bulk phase. This could be due to the difference of conformation adopted by these two molecules which could favor an aggregation when the anomeric form is $\beta \beta$. The interactions of the bolaform $\alpha \alpha$ with a model phospholipid, POPC, were investigated at the molecular level by infrared spectroscopy. Our results showed that these interactions involve the $\mathrm{C}=\mathrm{O}$ of the ester groups and the C-O-C bonds of the phospholipid. More detailed analyses of this interaction involving the acyl chains of the phospholipid will be performed using a deuterated phospholipid in order to discriminate them from the bolaform hydrophobic chain. This work offers a non-food valorizations of pentoses (xyloses) extracted from lignocellulosic biomass as surfactants, therapeutic or cosmetic agents for encapsulation.

\section{Acknowledgements}

M.N.N thanks the University of Liege for his postdoctoral position. M.D. and L.L. thanks the Belgian National Foundation for Scientific Research (FNRS) for their positions as Research Associate and Senior Research Associate, respectively. K. N. thanks the Superzym ARC grant, financed by the French Community of Belgium.

The authors thank the Walloon Region (through the Excellence Program «TECHNOSE ») for the financial support.

\section{References}

1. M. Deleu, C. Damez, S. Gatard, K. Nott, M. Paquot, S. Bouquillon, New J. Chem. 35, 2258 (2011) 
2. M. Deleu, S. Gatard, E. Payen, L. Lins, K. Nott, C. Flore, R. Thomas, M. Paquot, S. Bouquillon, C. R. Chimie 15, 68 (2012).

3. M.N. Nasir, A. Thawani, A. Kouzayha, F. Besson, Colloids Surf. B Biointerfaces 78, 17 (2010)

4. S. Dai, K.C. Tam, Colloids Surf. A 229, 157 (2003).

5. M.R. Wenk, J. Seelig, J. Phys. Chem. 101, 5224 (1997).

6. H. Heerklotz, J. Seelig, Biophys. J. 78, 2435 (2000).

7. H. Akutsu, Y. Kyoguku, H. Nakahara, K. Fukuda, Chem. Phys. Lipids 15, 222 (1975).

8. L. Ter-Minassian-Saraga, E. Okamura, J. Umemura, T. Takenaka, BBA 946, 417 (1988). 